\title{
The Luxury of Lockdown
}

\author{
Mehdi Shiva $^{1}$ (D) Hassan Molana ${ }^{2}$
}

Accepted: 13 March 2021 / Published online: 9 April 2021

(c) European Association of Development Research and Training Institutes (EADI) 2021

\begin{abstract}
Regression analysis based on data from Oxford COVID-19 Government Response Tracker and the World Bank datasets for 169 countries suggests that containment policies have, in general, a significant role in reducing the pandemic's fatality rate across all countries. However (i) there is at least a three weeks lag in realising the impact, (ii) the effectiveness increases with per capita income and, more specifically, (iii) workplace closure is ineffective in low-income countries. The analysis of data from Demographic and Health Survey (the DHS Programme) and IMF Policy Tracker indicates that developing countries are unlikely to have the basis required for effectively adopting stringent lockdown measures and instead would need to consider specifically targeted lockdown policies.
\end{abstract}

Keywords COVID-19 · Containment policies · Lockdown · Low-income countries

JEL Classification $\mathrm{H} 12 \cdot \mathrm{I} 18$

\section{Resume}

L'analyse de régression réalisée sur des données issues du panel de données de l'étude Oxford COVID-19 sur le suivi de la riposte gouvernementale et du panel de données de la Banque mondiale pour 169 pays suggère que les politiques de confinement ont, en général, un rôle important dans la réduction du taux de mortalité lié à la pandémie dans tous les pays. Cependant (i) il y a au moins trois semaines de délai dans l'apparition de l'impact, (ii) l'efficacité augmente avec le revenu par habitant et, plus précisément, (iii) la fermeture des lieux de travail est inefficace dans les pays à faible revenu. L'analyse des données de l'Enquête Démographique et de Santé (le programme EDS) et du Suivi des politiques du FMI indique qu'il est peu probable que les pays en développement disposent des bases nécessaires pour adopter efficacement des mesures de confinement strict et devraient plutôt envisager des politiques de confinement localisé.

Mehdi Shiva

mehdi.shiva@bsg.ox.ac.uk

1 Blavatnik School of Government, University of Oxford, 120 Walton Street, Oxford OX2 6GG, UK

2 School of Social Sciences, University of Dundee, Dundee DD1 4NH, UK 


\section{Introduction}

COVID-19 is a highly infectious disease because coronavirus spreads with relative ease. It is contagious even when carriers are asymptomatic and, in the early stages of the epidemic, on average one carrier can pass on the virus to more than two people (Sanche, 2020). The current pandemic has led to widespread morbidity and mortality as well as causing severe disruption of social, political and economic aspects of daily life. Governments around the world have responded with unprecedented measures, imposing strict social distancing and lockdown rules, to suppress the spread of the virus. The early evidence suggests that, on the whole, these policies have helped in limiting the spread of the virus (Hartl et al., 2020; Castex et al., 2020).

Disease mitigation strategies require informed decision-making to ensure a balance between the protection of the vulnerable from disease and the maintenance of global economic activity. There are two essential actions involved in dealing with the pandemic in a country: (i) controlling the spread of the disease, and (ii) caring for the patients (Shiva, 2020). While these two actions are interdependent and should be taken simultaneously, the first is directly managed by the policy authorities and involves the design and implementation of containment policies (and managing their impact on the economy), whereas the second falls on the country's health care system (over and above its normal daily service provision). A country's ability and readiness to cope with extreme lockdown measures dictate the nature of containment policies it can accommodate, and the existing capacity of its health care system and the speed with which it can be expanded determine the extent to which it can care for pandemic patients while providing its routine services.

In deciding to impose strict social distancing and lockdown rules, governments assess a trade-off between maintaining a certain level of economic activity and the costs of a rapidly spreading virus. The aim of these highly restraining policies is to minimise the spread of the virus and reduce subsequent fatalities, but their successful implementation requires the ability to put in place practical policies that help preserve people's livelihood. In advanced economies this is feasible given resources at their disposal and, in fact, governments in high-income countries have put in place extreme fiscal (and monetary) policy measures to strengthen the public health services and to mitigate economic and social consequences of such policies. But less developed and developing countries face a very different situation due to severe budget constraints, weak infrastructure, limited health care capacity, relatively large share of the informal economy, overcrowded towns and similar development-related factors. Despite this, many such countries also chose to enforce relatively stringent lockdown measures. There is already an emerging literature whose findings casts doubt on the effectiveness of lockdown measures in low-income countries. Amongst the existing studies, IGC (2020), Glassman et al. (2020) and Dercon (2020) provide evidence based on mainly descriptive analysis of the available information, while Castex et al. (2020) use statistical methods to quantify the impact of lockdown policies on disease transmission 
in different countries. Overall, there is evidence that per capita income, health expenditure and trained medical staff, as well as population density and size, are likely to play a role in determining the effectiveness of underlying policies.

In this paper, we have used data from 169 countries to investigate the impact of containment policies on fatalities on a global scale and have examined the extent to which their effectiveness across countries varies with their per capita income. Our regression analysis suggests that containment policies have a significant direct effect in reducing the fatality rate across all countries, beyond that achieved via reducing rate of infection. But we also find that (i) in general it takes at least three weeks for this effect to be realised, (ii) the effectiveness increases with per capita income and, more specifically, (iii) in low-income countries workplace closure is completely ineffective. The latter finding is also supported by our feasibility analysis which shows that low-income and lower-middle income countries are unlikely to have the minimum basis needed for effectively adopting stringent lockdown measures such as workplace closure. The sources of data used in this paper are briefly described in "Data", "The impact of containment policies" sections explains our statistical methodology and reports the results of our regression analysis, "Feasibility of containment policies" section considers the feasibility of containment policies in three countries chosen from different per capita income groups, and "Summary and conclusion" section concludes the paper.

\section{Data}

The main dataset used in our regression analysis is the Oxford COVID-19 Government Response Tracker (OxCGRT). This is a panel dataset which records-starting on 1 January 2020, for 169 countries and on a daily basis-information on a range of government responses to the crisis, including closures of education establishments (all primary and secondary schools and colleges and universities); closures of non-essential workplaces; cancellations of public events; restrictions on gatherings; closures of public transports; campaigns for informing the public; staying at home restrictions; and domestic and international travel restrictions. Hale et al. (2020) have used these nine indicators to construct a stringency index which varies between 0 and 100 and reflects the strictness of government policies implemented to reduce the chance of being exposed to the virus and hence to reduce the number of infected individuals and subsequent fatalities. This dataset also records daily infection and mortality statistics across the countries. In addition, in order to take account of country-specific characteristics - such as level of per capita income, per capita expenditure on health and population-in our analysis, we make use of the World Bank databank (WB, 2020).

The summary statistics for the relevant variables are provided in Tables 1 and 2. Table 1 shows that there is a clear difference between countries when they are classified into the four per capita income groups as suggested by the World Bank (low, lower-middle, upper-middle and high). These data are also presented differently in Fig. 1 to highlight a relative comparison and stress the fact that poorer countries have, on average, a larger population size that is much younger, with more 


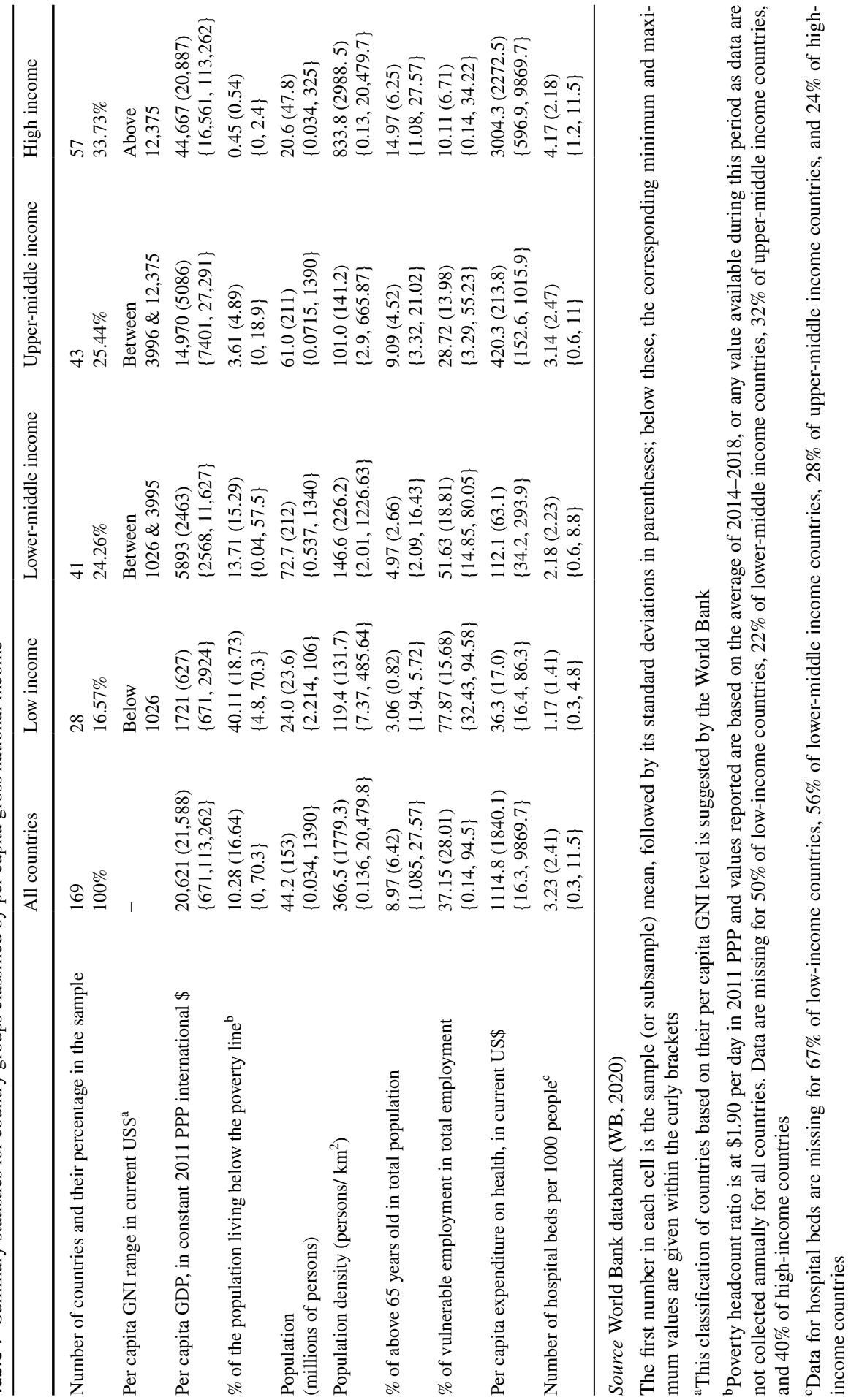
站 


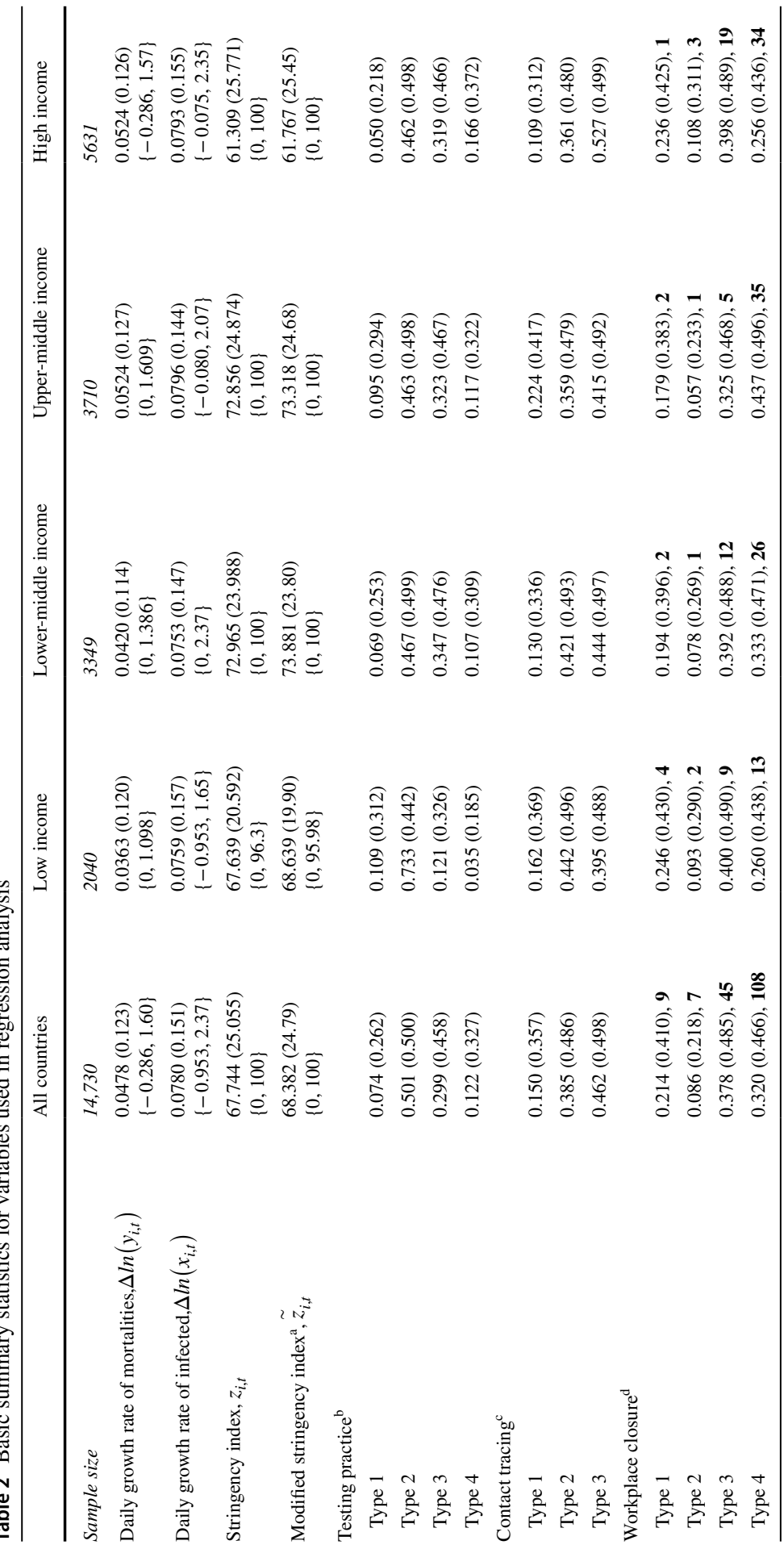




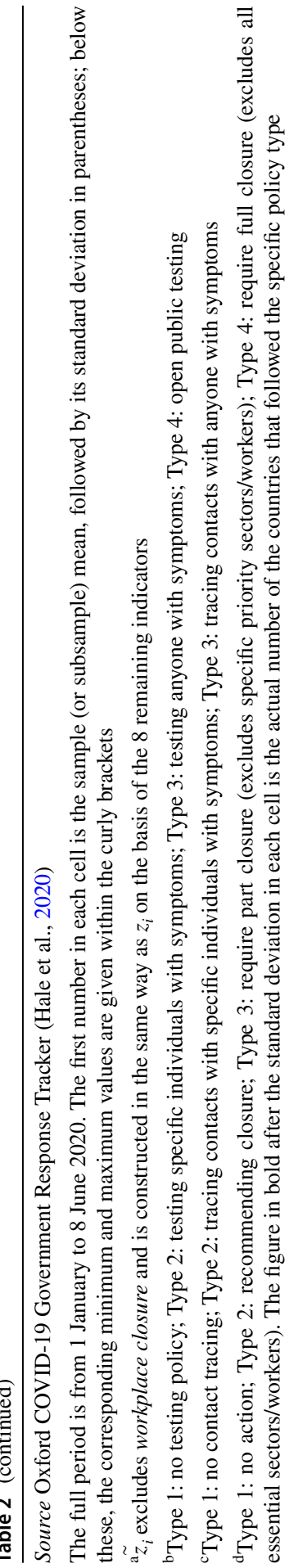

我造 


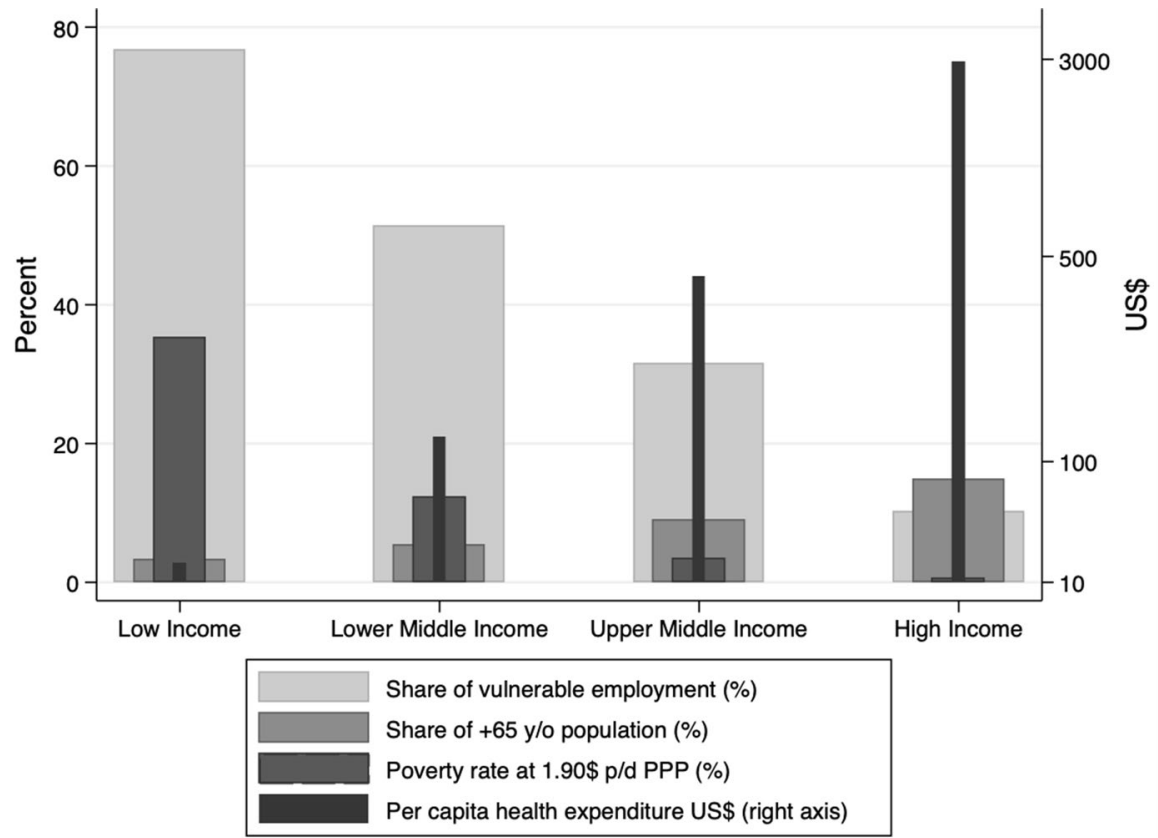

Fig. 1 Selected characteristics of countries that enforced (required) workplace closure

vulnerable employment and relatively very limited health care provision. Nevertheless, as Table 2 reveals, the average infection and mortality rates in poorer countries happen to be below that of the rest. The reason for this seems to lie, at least partly, in their approach to implementing the containment policies: they score an average stringency index which is above that of high-income countries and has a lower variability and, while their activities on testing practice and contact tracing are relatively lower, they appear to have followed a relatively strict workplace closure practice. This conclusion, as well as those derived from our regression analysis in the next section, is based on the assumption that data quality and testing intensity are not very different across the countries in the sample and should therefore be qualified by taking account the fact poorer countries face sever challenges in both respects.

It is worth noting that amongst the ingredients of the index 'workplace closure' is probably the strictest, and is likely to be more effective since it drastically reduces physical contacts outside the household. ${ }^{1}$ The recorded data show that, on average, 23 days after the outbreak most of the countries in the sample (109 out of 169) had shut down

\footnotetext{
1 For example, Ferguson et al. (2020) find that school closure is suggested to be insufficient to mitigate or supress an epidemic in isolation and the WHO-China (2020) Joint Mission report suggests that $80 \%$ of transmissions were likely to have occurred within the households that did not stay in.
} 
non-essential businesses. There are, however, good reasons to question the effectiveness of this policy in low-income countries. For instance, according to ILO criteria, a large part of employment in these countries can be classified as informal with no binding work contracts and protection of workers' rights ${ }^{2}$ - based on the existing estimates, this figure is about $80 \%$ of total employment. In such settings, recommending closure would simply not work while requiring closure would most likely put the workers and their families in great difficulty, therefore forcing them to find ways of bypassing the restrictions. It is also likely that closing markets and non-essential businesses, limiting harvesting and restricting movements push most households into further severe poverty and result in famine. This is particularly worrying since on average about $40 \%$ of the population live below the poverty line. In the light of this, our aim in "The impact of containment policies" section is to examine how the effectiveness of policies differs across groups of countries with different income groups.

In our case studies in "Feasibility of containment policies" section, we focus on the feasibility of lockdown and used data from the following datasets to shed further light on the actual situation in representative countries within each group:

(i) The Demographic and Health Survey (DHS) programme collects individual and household level data on population, health, HIV, and nutrition through more than 300 surveys across 90 countries (ICF, 2020).

(ii) IMF Policy Tracker (IMF, 2020) compiles key national policy responses to COVID-19 pandemic.

\section{The Impact of Containment Policies}

Denoting the value of stringency index of Hale et al. (2020) in country $i$ on day $t$ (the number of days since recording started) by $z_{i, t}$ and using $x_{i, t}$ to refer to the corresponding number of infected patients recorded over the period up to $t$, the following distributed lag regression equation could be used to estimate the overall impact of the ingredient policies on the rate of infection:

$$
\Delta \log \left(x_{i, t}\right)=\beta_{x}+\sum_{s=0}^{q} \alpha_{s} z_{i, t-s}+W_{i, t}^{\prime} \gamma+\varepsilon_{i, t},
$$

where $W_{i, t}$ is the vector of relevant country-level conditioning variables-such as introduction and nature of testing practice and existence of contact tracing that do not feature as components of $z$. $\alpha_{s}$ captures the impact of $z$ after $s$ days and we expect the total effect of $z$ on rate of change of $x$, i.e. $\sum_{s=0}^{q} \alpha_{s}$, to be negative where the maximum lag length $q$ is determined empirically. The regression Eq. (1) could be interpreted as a statistical, stochastic, generalisation of the basic version of the theoretical SIR model initially proposed by Kermack and McKendrick (1927). The

\footnotetext{
${ }^{2}$ ILO (2003) defines informal employment as comprising the total number of informal jobs, whether carried out in formal sector enterprises, informal sector enterprises, or households, during a given reference period.
} 
model is usually specified in continuous time $\tau$ and divides a constant population into three components consisting of susceptible (denoted by $S(\tau)$, are individuals who are exposed to infection), infected (denoted here by $x(\tau)$, are individuals who have already caught the disease and can transmit it to those who are susceptible-in the literature $I(\tau)$ is commonly used to refer to this class of individuals), and the rest (denoted here by $R(\tau)$, refers to those who have recovered or are resistant, and cannot catch or transmit the disease). $R(\tau)$ and $x(\tau)$ are assumed to evolve according the behavioural differential equations $\dot{R}=\gamma x$ and $\dot{x}=\beta S x-\dot{R}$ where a dot is placed over a variable to denote its derivative with respect to time, $\gamma$ and $\beta$ are constant positive coefficients, and the system of differential equations is completed by adding $\dot{x}+\dot{R}+\dot{S}=0$ which imposes the assumption of constancy of population. ${ }^{3}$ These behavioural equations imply $\frac{\dot{x}}{x}=\beta S-\gamma$ which in discrete time can be written as $\Delta \log \left(x_{t}\right)=\beta S_{t}-\gamma$. However, when $S_{t}$ is very large and can be treated as a constant (subject to unpredictable random deviations) relative to the rest of the variables and parameters involved - as is the case at the initial stage of a nationwide, large population, pandemic such as COVID-19-we can approximate $\beta S_{t}-\gamma$ by a positive constant coefficient, $\beta_{x}$ say, and use the regression equation $\Delta \log \left(x_{t}\right)=\beta_{x}+\varepsilon_{t}$ where the stochastic disturbance term $\varepsilon_{t}$ embodies the effect of all unrelated factors and the sample of observations for estimating $\beta_{x}$ is based on random draws from a given population. When the sample involves a panel consisting of a number of countries indexed by $i=1, \ldots, n$, which follow a set of containment policies with different emphasis and intensity and also differ in their main characteristics as explained in "Data" section, the regression ought to be conditioned on the relevant countyspecific factors as in (1) above so as to avoid introducing a bias in the regression residuals due to omitting shifts between countries. The estimated values of $\beta_{x}$ and $\alpha_{s}$ therefore reflect the average responses of the dependent variable to the corresponding explanatory variables across the countries within the sample.

Let $y_{i, t}$ denote the number of daily deaths recorded up to day $t$ in country $i$. The relationship between $x_{i, t}$ and $y_{i, t}$ can then be quantified by estimating the following distributed lag regression equation

$$
\Delta \log \left(y_{i, t}\right)=\beta_{y}+\sum_{s=0}^{p} \varphi_{s} \Delta \log \left(x_{i, t-n-s}\right)+V_{i, t}^{\prime} \vartheta+\xi_{i, t},
$$

where the dependent variable is a measure of fatality rate, $V_{i, t}$ is the vector of relevant country-specific conditioning variables-such as population's health condition, national health care provision, income, poverty and inequality measures that do not feature as components of $z$, but excludes those that are accounted for in the index $z$ - and it is assumed that there is a window of $p$ days, which start $n$ days after being infected, during which death from infection could occur-see Castex et al. (2020)

\footnotetext{
3 See Thompson (2020) for a discussion of using the SIR model to predict the evolution of COVID-19 infections in the UK and to develop a better understanding of decisions about the timing of policies. Rodrigues (2016) discusses the new developments in the application of SIR-type models and Giordano et al. (2020) develop and apply a generalised version of SIR, called SIDARTHE, which consists of 8 differential equations and use it to model the spread of COVID-19 in Italy.
} 
and Wagner et al. (2020) and note that in practice $p$ and $n$ can be allowed to be determined by data.

In principle, the effect of $z$ on $x$ as well as on $y$ could be worked out by estimating the above regression equations jointly. However, the fact that accurate data on $x$ are not available undermines the robustness of estimates obtained in this way and it is more efficient, and informative, for our purpose to directly estimate a regression equation that is based on combining Eqs. (1) and (2), which can be written as

$$
\Delta \log \left(y_{i, t}\right)=\theta+\sum_{s=0}^{q+p} \eta_{s} z_{i, t-n-s}+\Omega_{i, t}^{\prime} \delta+\sum_{s=l}^{k} \lambda_{s} \Delta \log \left(x_{i, t-s}\right)+u_{i, t},
$$

where $\Omega_{i, t}$ is the augmented country-specific characteristics vector which combines $V_{i, t}$ and $W_{i, t}, u_{i, t}$ is the augmented disturbance term, and $\Delta \log \left(x_{i, t-s}\right)$ are included to control for the effect of excess variations in the infection rate where the lag $l$ and duration $k$ are allowed to be determined by data so as to reduce any serial correlation in the residuals. ${ }^{4}$ The pattern of the estimated values of coefficients $\eta_{s}$ and their statistical significance should reveal the nature of the overall impact of policies in reducing fatality beyond that captured by infection rates and moderated by other country-specific variables.

The sample of observations used in estimating the regression Eq. (3) is based on combining OxCGRT and WB datasets explained in "Data" section. In order to determine the nature of the impact of $z_{i, t-n-s}$, we estimated (3) allowing for different lag structures and found that including more than one lag was problematic, mainly due to strong multicollinearity arising from the fact that the values of this index do not vary much from one day to the next. In Table 3, we report the coefficient estimates of the impact of the index based on including one lag only, denoted by $z_{i, t-m}$, for different lag length $m$. The statistical performance of the regression is best when $m$ is set to four weeks and the statistical significance of coefficient estimate of $z_{i, t-m}$, $\hat{\eta}_{m}$, also reaches its highest level at that lag. The value of $\hat{\eta}_{m}$ however is rather small and implies that, on average, a 10-unit increase in the index is associated with a reduction of around $1 \%$ in fatalities, once we have controlled for variations in infection rates and other relevant factors. In order to ensure that $\hat{\eta}_{m}$ is as robust as possible, when estimating Eq. (3) we also took into account the following:

- Since data on elements of $\Omega_{i}$ are not available in daily frequency, we replaced $\Omega_{i}$ with country dummies (set to unity for country $i$ and zero otherwise) and used the fixed effect estimator as in standard panel regressions.

- In the above specifications, we have assumed for simplicity that the effect of $z$ is linear but this need not necessarily be the case. Hence, we experimented with

\footnotetext{
4 Idiosyncratic residual serial correlation is likely to result in these regressions because the dependent variable is bound to exhibit a systematic pattern of fluctuation which is unlikely to be captured by the main explanatory variables which cannot, by definition, embody such fluctuations. The only variable which could have similar fluctuations to the dependent variable on the same frequency is the rate of growth of infections. Hence, adding the latter as explanatory variables increases the robustness of the estimates of coefficients of the stringency index.
} 
Table 3 Fixed effect estimates of coefficient estimate of $z_{i, t-m}$

\begin{tabular}{lcclll}
\hline Lag $m$ & Estimate of $\eta_{m}$ & $t$-ratio & Within $R^{2}$ & Between $R^{2}$ & Overall $R^{2}$ \\
\hline 1 week & 0.000615 & 3.57 & 0.0996 & 0.0043 & 0.0822 \\
2 weeks & -0.00017 & -0.98 & 0.0963 & 0.0121 & 0.0882 \\
3 weeks & -0.00101 & -8.65 & 0.109 & 0.0556 & 0.1023 \\
4 weeks & -0.00107 & -10.32 & 0.1117 & 0.1149 & 0.1087 \\
5 weeks & -0.00084 & -8.13 & 0.1062 & 0.1199 & 0.1067 \\
6 weeks & -0.00056 & -5.3 & 0.1013 & 0.0628 & 0.0982 \\
7 weeks & -0.00015 & -1.39 & 0.0994 & 0.0041 & 0.0882 \\
8 weeks & 0.000075 & 0.75 & 0.0977 & 0.0005 & 0.0841 \\
\hline
\end{tabular}

The estimates are based on Eq. (3). The regression equation for each row included only one $z_{i, t-m}$. The full period is from 1 January to 8 June 2020. The sample size with 8 weeks lag is 13756 . The $t$-ratios are based on standard errors clustered at the country level. In the interest of saving space, we only report the relevant coefficient estimates in Tables 3 and have provided the full set of estimates in the online Appen$\operatorname{dix}$

both quadratic and logarithmic specifications as well, to capture any non-linearity that might exist in the way the policy effect carries through, but opted for the linear specification after comparing the statistical performance of the underlying regressions.

- Because the pattern of time trend could differ across countries, we also tried including country-specific trend as well as a general time trend term. To capture any non-linearity in trend, we allowed for a cubic trend component.

- Given that data consist of daily observations and there is likely to be a systematic difference in the way individuals react to policies, for instance due to their cultural habits, we introduced week-day dummies to capture any daily variations unexplained by other factors.

- Finally, we also tried including a general time fixed effect to capture any daily shifts-on average across all countries-which other factors cannot account for.

Having found a significant effect associated with the stringency index, we now turn to our main objective of testing whether this effect is homogenous across countries with different per capita income. This is particularly motivated by current debates on the effectiveness of containment measures in lower-income countries (Castex et al., 2020; Ray and Subramanian, 2020; Baldwin, 2020; Nemzoff et al., 2020). To carry out the test, we used the income classification reported in Table 1 and created country-type dummy variables $I G_{i, t}^{j}$ to represent income groups, where the $j=0,1,2,3$, respectively, represents 'low income', 'lower-middle income', 'upper-middle income', and 'high income'; thus, $I G_{i, t}^{j}=1$ if country $i$ is within income group $j$ and $I G_{i, t}^{j}=0$ otherwise. We augmented the previously estimated regression equation with these dummies and their interaction with $z_{i, t-m}$, therefore using the following modified version of regression Eq. (3), 


$$
\Delta \log \left(y_{i, t}\right)=\theta+\eta_{m} z_{i, t-m}+\sum_{j=1}^{3}\left(\mu_{j}+\rho_{j} z_{i, t-m}\right) I G_{i, t}^{j}+\mathrm{H}_{i, t}^{\prime} \pi+\omega_{i, t},
$$

where, based on the previous results, we set the lag length $m$ to 4 weeks. The vector $H_{i, t}$ comprises the rest of the (control or conditioning) explanatory variables as explained above and $\omega_{i, t}$ is the random disturbance term. We have excluded $I G_{i, t}^{0}$ and used the low-income group as the base group. The estimated effects of the index, based on panel fixed effect estimation of (4), reveal that the impact of $z$ remains statistically significant but the corresponding average marginal effects (AMEs), represented by $\hat{\eta}_{m}+\hat{\rho}_{j} I G_{i, t}^{j}$ which are reported in Table 4 , imply that containment policies are more effective the higher is per capita income; $\hat{\eta}_{m}+\hat{\rho}_{j} I G_{i, t}^{j}$ for the low and lower-middle income groups is significantly smaller than those of the upper-middle and high-income groups. Put differently, our data do not support the hypothesis that containment measures affect fatality rates similarly across all countries, regardless of their per capita income levels.

To put our empirical finding in a more relevant context, it is worth noting that one of the main reasons for introducing containment measures is to slow down the speed of transmission so as to allow sufficient time for a health service system with a given capacity to prepare for caring for COVID patients with critical and severe symptoms while maintaining a target level for its regular services to non-COVID patients-see Shiva (2020). In this sense, therefore, one could argue that the ultimate effect of containment measures is to reduce COVID-related fatalities and the containment policy could be seen as strategically essential in a country which can afford it. But this interpretation is not particularly relevant in a typical poor country which faces serious challenges in imposing effective social distancing, have alreadyoverstretched hospitals and very limited capacity to rapidly scale up health care provision. For example, a recent survey of 47 African countries undertaken by WHO (2020) revealed that there were on average nine intensive care unit beds per one million people; Roberton et al. (2020) estimate that a reduction in coverage of basic life-saving interventions of $15 \%$ for 6 months in low and lower-middle income countries is likely to lead to additional 253,500 child deaths and 12,190 maternal deaths; Maclean and Marks (2020) calculate that there are fewer than 2000 working ventilator units to serve hundreds of millions of people in public hospitals across 41 African countries, compared with more than 170,000 units in the US; and Ismail et al. (2020) and Smith et al. (2020) find that investing in oxygen equipment instead of ventilators or ICU rooms is a more cost-effective strategy and a much quicker option in low- and middle-income countries.

Given that the stringency index represents the weighted average of a range of different policies, it is also important to explore the difference in the impact of its main ingredients across countries. As explained in "Data" section, amongst these ingredients workplace closure is probably the most effective measure and was adopted, on average, at the same scale by lower-income countries but there are good reasons to doubt its sustained effectiveness in lower-income countries. However, as can be seen from Table 2, workplace closure data used in constructing the stringency index consist of four types-'no closure', 'recommend closure', 'require part closure' 
Table 4 Fixed effect estimates of the average marginal effects of stringency index at different per capita income levels

\begin{tabular}{llllll}
\hline Income groups & Estimate & St. Error & $z$ Score & \multicolumn{2}{c}{ 95\% Confidence interval } \\
\hline Low Income & -.0008077 & .0001512 & -5.34 & {$[-.0011041$} & $-.0005113]$ \\
Lower-middle income & -.0008242 & .0001033 & -7.98 & {$[-.0010267$} & $-.0006218]$ \\
Upper-middle income & -.0012016 & .000112 & -10.73 & {$[-.0014211$} & $-.0009821]$ \\
High income & -.001331 & .0001309 & -10.17 & {$[-.0015876$} & $-.0010744]$ \\
\hline
\end{tabular}

Estimates are based on Eq. (4) with 4 weeks lag and the standard errors are clustered at the country level. The sample size is 14591

(except some sectors or categories of workers) and 'require full closure' (except essential workers) - and it is important to distinguish between these when checking the role of the policy. To investigate this, and to quantify the extent to which impacts of closure policies vary across countries within different income groups, we identify, and control for, countries in different income groups adopting one or more of the underlying policies. Consider the dummy variables $L D_{i, t}^{c}$ where the superscript $c=0$ corresponds to the base policy of 'no closure' and $c=1,2,3$, respectively, represent 'recommend closure', 'require part closure', and 'require full closure'; thus, $L D_{i, t}^{c}=1$ when country $i$ has adopted policy $c$ and $L D_{i, t}^{c}=0$ otherwise. These dummies together with $I G_{i, t}^{j}$ income group dummies, and their interactions, are then included in the regression equation, forming an augmented version of Eq. (4) that enables capturing the role of specific policies in different country groups. However, since $L D_{i, t}^{c}$ dummies pick up the effect of workplace closure component of the stringency index $z_{i}$, we replace it with a new index, $z_{i}$, which excludes workplace closure and is based on a weighted average of the remaining eight policies instead (see Table 2 for a basic comparison of $z_{i}$ and $z_{i}$ ), and therefore estimate the regression equation

$$
\begin{aligned}
\Delta \log \left(y_{i, t}\right) & =\theta+\tilde{\eta}_{m} \tilde{z}_{i, t-m}+\sum_{j=1}^{3}\left(\tilde{\mu}_{j}+\tilde{\rho}_{j} \tilde{z}_{i, t-m}\right) \mathrm{IG}_{i, t}^{j} \\
& +\sum_{c=1}^{3}\left(\varphi_{j}+\sigma_{j, m} \tilde{z}_{i, t-m}\right) \mathrm{LD}_{i, t}^{c}+H_{i, t}^{\prime} \tilde{\vartheta}+\tilde{\omega}_{i, t},
\end{aligned}
$$

where we have put a $\varepsilon \sim \varepsilon$ over $\eta, \mu, \rho, \vartheta$ and $\omega$ to distinguish them from those in Eq. (4). Table 5 reports the values of the relevant AMEs based on coefficient estimates of Eq. (5) obtained with the lag length $m$ fixed at 4 weeks. These support our conjecture that the impact of workplace closure on fatalities is not universal and depends on a country's economic development level, here approximated by its per capita income: a total shutdown of workplace is effective in all but low-income countries where all types of work closure policies are found to be statistically insignificant. In order to ensure that these results are not specific to fixing the lag length 
$m$ at 4 weeks, we also checked the AMEs obtained when it is set to 3, 5, 6 or 7 weeks which supported the same conclusion. ${ }^{5}$

Our findings are consistent with the narrative in the related literature that questions the usefulness of the strategy when a country's policy authorities decide to emulate other countries' approach in dealing with the pandemic. When policies are not modified to take account of the country's economic strength, well-being, welfare provision and indigenous cultural characteristics, people are likely to either ignore them or find ways around them, rendering the policies ineffective. Baldwin (2020) warns that "if the implied wellbeing losses involve hunger and/or outrageous inequalities, the intolerance could manifest itself quickly and chaotically" (p. 1).

Our results also highlight the importance of using 'smart' or 'targeted' lockdown policies on the grounds that country-specific conditions call for designing particular policies that work better given underlying circumstances. An example of a targeted policy for India is outlined in Ray and Subramanian (2020), where they argue against a comprehensive lockdown and propose their 'reasonable alternative' whereby young adults are allowed to work while elderly members of households are insulated and cared for by their own families. Ferguson et al. (2020) explain that targeted social distancing such as putting in place more stringent measures for the elderly might be a more appropriate strategy even in high-income countries. Glassman et al. (2020), IGC (2020) and IASC (2020) further provide practical guidance for a range of alternative realistic pandemic policy responses for low-income countries and Broadbent et al. (2020) develop and propose a customisable framework and policy decision tool. In "Feasibility of containment policies" section, therefore, we try to illustrate below by example how a basic feasibility assessment can reveal useful information on identifying and targeting a policy in different countries.

\section{Feasibility of Containment Policies}

There are two main requirements, or necessary conditions, for successfully implementing a lockdown policy which imposes strict home isolation to achieve maximum social distancing via reducing, as much as possible, contacts with non-family members: (i) state's ability to enforce the underlying rules; and (ii) households' actual capacity to abide by those rules. Of these, clearly, the latter is more problematic since blanket imposition assumes that all households can tolerate the economic and psychological pressures that rapidly develop in such situations. In fact, the risks involved are considerable and policy authorities are, in general, keen to have an assessment of their feasibility in this context. However, it is understood that these risks are very likely to be amplified in low-income countries which face a severe

\footnotetext{
5 These results are not reported but are available from the authors on request. As a referee points out, these results and conclusions disregard the fact that some workers were already working from home before the policy was implemented, and that the fraction of the labour force working remotely is likely to be higher in high-income countries. In the absence of data, we hope that country fixed effects and country income group dummies at least partially correct for the impact of such discrepancies.
} 
Table 5 Estimates of the average marginal effects of the stringency index at different per capita income levels

\begin{tabular}{clllll}
\hline & Estimate & St. Error & $z$ Score & 95\% Confidence interval \\
\hline Low income & & & & & \\
Type 2: recommend closure & -0.007437 & 0.02514 & -0.30 & {$[-0.0567105$} & $0.0418364]$ \\
Type 3: require part closure & -0.0290175 & 0.0158845 & -1.83 & {$[-0.0601506$} & $0.0021156]$ \\
Type 4: require full closure & -0.0251344 & 0.0152981 & -1.64 & {$[-0.0551181$} & $0.0048493]$ \\
Lower-middle income & & & & & \\
Type 2: recommend closure & -0.0115163 & 0.0193542 & -0.60 & {$[-0.0494499$} & $0.0264172]$ \\
Type 3: require part closure & -0.0303993 & 0.0132559 & -2.29 & {$[-0.0563803$} & $-0.0044183]$ \\
Type 4: require full closure & -0.0385359 & 0.0135953 & -2.83 & {$[-0.0651822$} & $-0.0118896]$ \\
Upper-middle income & & & & & \\
Type 2: recommend closure & -0.0108084 & 0.0103968 & -1.04 & {$[-0.0311857$} & $0.009569]$ \\
Type 3: require part closure & -0.0381196 & 0.0118442 & -3.22 & {$[-0.0613337$} & $-0.0149055]$ \\
Type 4: require full closure & -0.0344651 & 0.0094437 & -3.65 & -0.0529744 & $-0.0159559]$ \\
High income & & & & & \\
Type 2: recommend closure & -0.0190401 & 0.0112498 & -1.69 & {$[-0.0410894$} & $0.0030092]$ \\
Type 3: require part closure & -0.0287052 & 0.0091373 & -3.14 & {$[-0.0466139$} & $-0.0107965]$ \\
Type 4: require full closure & -0.0438838 & 0.0116527 & -3.77 & {$[-0.0667227$} & $-0.021045]$ \\
\hline
\end{tabular}

Estimates are based on Eq. (5) with 4 weeks lag and the standard errors are clustered at the country level. The sample size is 14591

shortage of hygiene farcicalities-e.g. in 2015, only $15 \%$ of sub-Saharan Africans had access to basic hand-washing facilities (Unicef, 2020)—and most households have extremely limited savings and liquidity so it is conceivable that a rapid rise in unemployment and a considerable loss of income can lead to food insecurity, malnutrition and starvation.

To highlight developing countries' readiness for a lockdown and provide an idea of practicality of the underlying policies, we follow the methodology introduced by Jones et al. (2020) and use three case studies-one from each of lowincome, lower-middle income and upper-middle income groups-to illustrate the relative extent to which countries in different income groups face such problems. We would like to emphasise at the outset that our analysis below is merely illustrative and to this end we have chosen Afghanistan, Nigeria and Colombia on the basis of their income group, respectively, their location (one from each continent), some similarity in their main demographic properties, and availability of more recent data, and the fact that in response to the pandemic these countries have enforced a lockdown policy, including work place closure, for a period of time.

The survey data from the DHS programme provide information on the indicators described by Jones et al. (2020), namely households' access to Electricity; Clean Drinking Water; Improved Sanitation; Telephone (landline or mobile); and 
Regular or Formal Employment. ${ }^{6}$ A summary of our findings for the three countries chosen is presented in Table 6. Combining this information with IMF policy tracker enables us to compare governments' response to the pandemic given the readiness of households.

Our case studies analysis suggests that all three governments tried to mitigate the pressure on vulnerable households by introducing a variety of protective policies. However, the level of support and number of people who benefit from such measures depend on state's capacity.

In Afghanistan, there is a support programme that provides free bread to needy families, but its coverage is limited to a very small portion of households in the whole country (Saif, 2020). 90\% of the population in the country (around 30 million people) do not have access to electricity, clean water, or improved sanitation (based on 2015 estimates) and most people need to go out of their house for their basic needs. This is especially prevalent in rural areas where only about $1 \%$ of the population have access to all the above-mentioned necessities. In addition, given the state's limited capacity, it is very unlikely that those who need support can receive it in good time. As Dercon (2020) explains, even though rural population is relatively less prepared, those working in informal sector in urban areas and non-farm sector in rural regions are likely to be in a worse situation in this respect. It is worth mentioning that $94 \%$ of the country's population is not involved in regular or formal employment. Thus, enforcing a lockdown policy might have severe consequences, as could be seen by the increased number of Afghan families falling into poverty since the initiation of lockdown. Oxfam (2020) estimates that the number of people on the brink of famine in Afghanistan has risen substantially just after a month into the lockdown.

In Nigeria, where the formal employment rate is substantially higher than Afghanistan-51\% compared to $6 \%$ - people on average enjoy a slightly better economic condition. But access to clean drinking water and electricity, which is even lower than in Afghanistan, remains a serious challenge; e.g. approximately only one in five of urban residents and one in ten of rural residents have access to clean drinking water in their premises. This portrays a similar survival situation; most people (89\%), especially in rural areas (99\%), need to go out of their house for their basic needs and it is very unlikely that those who need support can receive it in good time. At the time of writing this text, three major states in the country are under lockdown and there is a nationwide night-time curfew. There are some concerns with imposing lockdown in densely populated cities such as Lagos since, instead of social distancing, this might force a large number of people to be crammed in a single room in poor sanitary conditions and without easy access to most basic hygiene facilities. Despite the sharp decline in oil prices,

\footnotetext{
6 Two examples from the existing studies that have used this method are Jones et al. (2020) for Mozambique who conclude that it would only be feasible to immediately lock down around $6 \%$ of all households, that only $13 \%$ of all households are partially ready for lockdown since they have access to basic services but lack the required financial capacity, and that the rest are totally unprepared; Nemzoff et al. (2020) for Bolivia who find that only $2.3 \%$ of households are fully ready for a lockdown while $44.8 \%$ are totally unprepared.
} 
Table 6 Lockdown readiness (the DHS programme)

\begin{tabular}{lllll}
\hline & & National (\%) & Rural (\%) & Urban (\%) \\
\hline Afghanistan & Electricity & 72 & 66 & 89 \\
& Clean drinking water & 25 & 17 & 48 \\
& Improved sanitation & 24 & 14 & 54 \\
& All three above & $\mathbf{1 0}$ & $\mathbf{1}$ & $\mathbf{3 0}$ \\
& Telephone (landline or mobile) & 86 & 84 & 99 \\
\multirow{5}{*}{ Nigeria } & Regular or Formal Employment & 6 & 6 & 7 \\
& Electricity & 55 & 38 & 80 \\
& Clean drinking water & 16 & 12 & 22 \\
& Improved sanitation & 51 & 37 & 71 \\
& All three above & $\mathbf{1 1}$ & $\mathbf{1}$ & $\mathbf{1 8}$ \\
& Telephone (landline or mobile) & 87 & 82 & 99 \\
& Regular or formal Employment & 51 & 46 & 60 \\
Colombia & Electricity & 96 & 86 & 99 \\
& Clean drinking water & 83 & 55 & 93 \\
& Improved Sanitation & 93 & 77 & 99 \\
& All three above & 80 & 48 & 92 \\
& Telephone (landline or mobile) & 95 & 89 & 99 \\
& Regular or formal employment & 54 & 40 & 58 \\
\hline
\end{tabular}

Source: DHS programme (ICF, 2020)

We used household and individual recodes. Afghanistan and Colombia data are based on 2015 survey, Nigeria based on 2018. Safe drinking water is defined as drinking water on premises from the public network, tube or protected well, or bottled mineral water. Improved sanitation is defined as flush toilet inside the household or compound or improved latrine. Regular or formal employment includes individuals who work all year and excludes seasonal and occasional workers. The legal working age is set to 16

which occurred at the same time as the pandemic and shrank the state's financial capacity, a number of expansionary monetary and fiscal policies were introduced to keep the impaired economy alive. However, a nationwide lockdown, especially if it includes work place closure, seems inappropriate given the low readiness of the population and their reliance on their jobs.

In Colombia, which is classified as an upper-middle income country, data suggest that most people are likely to survive stay-in conditions with respect to their basic needs; $80 \%$ of the population has access to the three above-mentioned necessities. Thus, unlike Afghanistan and Nigeria, it would be justified if the authorities paid some attention to secondary considerations such as income support for low-skill workers and provision of infrastructure to facilitate remote-working for high-skill workers. The government has in fact implemented measures to support the vulnerable via fiscal policies such as a payroll subsidy equivalent to $40 \%$ of the minimum wage per worker for specific businesses that have substantially suffered from the lockdown.

In comparison to the above groups of countries, in a typical high-income country there are two core considerations which feature at the forefront of any feasibility 
study. The first involves the extent to which households' main survival needsessential food, health and safety requirements-are met. Unless the provision is adequate, even a short-term lockdown will be ineffective as household members will need to make multiple daily trips outside their home. In the UK, for example, ONS (2020) records that almost all residents have access to electricity and running water, around $99 \%$ enjoy standard sanitation, telephone coverage is comprehensive and about $95 \%$ of adults have access to internet. Therefore, the problem is mainly a matter of providing adequate protection for the most vulnerable for their safety, security, and loss of income and jobs to minimise threats of falling into perpetual poverty trap. This brings us to the second consideration, which concerns the fiscal sustainability of financial compensation policies which is a considerable challenge in a situation where production and trade have substantially reduced and most of low-skill workers as well as a considerable proportion of high-skill workers are not able to work. In a study of the EU countries, Midoes (2020) finds that 99 million individuals live in households whose savings would not cover their most basic expenses (e.g. food, utilities and rent/mortgage) beyond two months. Clearly, any commitment to protect incomes and jobs of these individuals will require a considerable shift in fiscal policy in high-income countries where, as Ferguson et al. (2020) observe, "no public health intervention with such disruptive effects on society has been previously attempted for such a long duration of time. How populations and societies will respond remains unclear" (p. 16).

\section{Summary and Conclusion}

Using the OxCGRT and the World Bank datasets, our regression analysis reveals that containment policies have a significant direct effect in reducing the fatality rate of COVID-19 pandemic. However, there is at least a three weeks lag in realising the impact. We also find that the effectiveness of these policies reduces across countries with per capita income, and that workplace closure is likely to be completely ineffective in low-income countries. This is due to two inefficiencies, (a) weak state capacity, and (b) unreadiness of people to abide by the restrictive rules. The former refers to low health and financial capacity, and poor infrastructure. For instance, even if we manage to deliver more ventilators to the least-developed countries across Africa, there are neither enough trained medical personnel who could utilise the machines, nor reliable electricity supply and piped oxygen facilities. The presence of these basic considerations is taken for granted in developed countries' hospitals, but they are frequently absent in health facilities across low-income countries. Also, most developing-country governments have large levels of external debt, low tax revenues, and high credit risk which makes it difficult for them to borrow (Loayza and Pennings, 2020), hence undermining the feasibility of enforcing lockdown policies that cannot be accompanied by adequate financial easing to facilitate stay-in conditions. Our case studies, based on data from DHS surveys and descriptive information from IMF Policy Tracker for Afghanistan, Nigeria and Colombiawhich are chosen to demonstrate the situation of countries from different income 
groups-imply that readiness to implement lockdown measures varies drastically across developing countries.

It is worthwhile stressing that even those households that have adequate access to the infrastructure to satisfy their basic needs in-house face financing problems. Whereas most of the high-skill workers would be able to maintain their income if they could carry on with their work remotely, low-skilled workers whose jobs require physical presence at workplace are likely to struggle, especially in developing countries where a large portion such of jobs are informal. Various measures are being taken across the world to make sure that formal sector workers are not laid off and retain some of their earnings. Nevertheless, even rich economies struggle to provide financial support to casual workers and the self-employed. This dire situation calls for pragmatic and effective solutions, tailored to the reality of poor and developing countries.

On the whole, our evidence highlights the importance of using 'smart' or 'targeted' approach to imposing lockdown measures for developing countries as long as they are tailored for the local conditions and do not blindly emulate those recommended mainly for high-income countries. While there is evidently no 'one-size fits all' response to this worldwide crisis, the unprecedented nature of the situation calls for a rapid learning of effective measures (Shiva, 2020) so as to minimise stress and casualties across different countries; Dercon (2020) identifies these as 'no-regret policies' which are effective but carry minimal risk.

Supplementary Information The online version contains supplementary material available at https://doi. org/10.1057/s41287-021-00389-x.

Acknowledgements We are grateful to Kalipso Chalkidou, Jeremy Boccanfuso, Adrian Gheorghe and three anonymous referees for helpful comments and suggestions.

Funding No funding was received for this study.

\section{Compliance with Ethical Standards}

Conflict of interest On behalf of all authors, the corresponding author states that there is no conflict of interest.

\section{References}

Baldwin, R. 2020. The COVID-19 upheaval scenario: Inequality and pandemic make an explosive mix. VoxEU.org, March 15, 2020.

Broadbent, A., A. Kaziboni, B. Smart, O. Tella, and D. Walker. 2020). A framework for decisions in a postCOVID world. Institute for the Future of Knowledge.

Castex, G., E. Dechter, and M. Lorca. 2020. COVID-19: Cross-country heterogeneity in effectiveness of non-pharmaceutical interventions. COVID Economics, Vetted and Real-Time Papers Issue 14, May 6, 2020.

Dercon, S. 2020. No-Regret Policies for the COVID-19 Crisis in Developing Countries. Washington: Centre for Global Development.

Ferguson, N., D. Laydon, G. Nedjati Gilani, N. Imai, K. Ainslie, M. Baguelin, and A. Dighe. 2020. Report 9: Impact of non-pharmaceutical interventions (NPIs) to reduce COVID-19 mortality and healthcare demand. 
Giordano, G., F. Blanchini, R. Bruno, et al. 2020. Modelling the COVID-19 epidemic and implementation of population-wide interventions in Italy. Nature Medicine 26: 855-860. https://doi.org/10.1038/ s41591-020-0883-7.

Glassman, A., K. Chalkidou, and R. Sullivan. 2020. Does one size fit all? Realistic alternatives for COVID19 response in low income countries. Washington: Centre for Global Development.

Hale, T., A. Petherick, T. Phillips, and S. Webster. 2020. Variation in government responses to COVID19. Blavatnik School of Government Working Paper, 31.

Hartl, T, K. Waelde, and E. Weber. 2020. Measuring the impact of the German public shutdown on the spread of COVID-19. COVID Economics, Vetted and Real-Time Papers Issue 1, April 5, 2020.

IASC. 2020. Public health and social measures for COVID-19 preparedness and response in low capacity and humanitarian settings. Geneva: Inter-Agency Standing Committee.

ICF. 2020. Demographic and Health Surveys (various) [Afghanistan, 2015; Nigeria, 2018; Columbia, 2015]. Funded by USAID. Rockville, Maryland: ICF.

IGC. 2020. IGC COVID-19 guidance note: Containment strategies and support for vulnerable households. London: The International Growth Centre.

IMF. 2020. Policy responses to COVID-19. International Monetary Fund, https://www.imf.org/en/Topics/ imf-and-covid19/Policy-Responses-to-COVID-19

ILO. 2003. Report of the conference: Seventeenth international conference of labour statisticians. Geneva: International Labour Organisation.

Ismail, I., T. Baker, P. Baker, K. Chalkidou, Y. Chi, and R. Sullivan. 2020. Strengthening the basics: Approaches to COVID-19 care in low-resource settings. Washington: Centre for Global Development.

Jones, S., E.M. Egger, and R. Santos. 2020. The five criteria low income countries must have in place for lockdowns to work. Helsinki: United Nations University UNU-WIDER.

Kermack, W.O., and A.G. McKendrick. 1927. A contribution to the mathematical theory of epidemics. Proceedings Royal Soc. London (A) 115: 700-721.

Loayza, N. V., and Pennings, S. 2020. Macroeconomic policy in the time of COVID-19: A primer for developing countries. Research and Policy Briefs No 28. World Bank Group.

Maclean, R., and S. Marks. 2020. 10 African Countries Have No Ventilators. That's Only Part of the Problem. New York Times. April 18, 2020.

Midoes, C. 2020. Who can live without two months of income? COVID Economics, Vetted and Real-Time Papers 18, May 15, 2020.

Nemzoff, C., K. Chalkidou, and D. Walker. 2020. Are strict lockdowns suited to populations who live hand to mouth? A Closer Look at Malawi. Washington: Centre for Global Development.

ONS. 2020. Internet access-households and individuals. Newport: Office for National Statistics.

Oxfam (2020). The hunger virus: How COVID-19 is fuelling hunger in a hungry world. Oxfam policy papers. Oxfam International.

Ray, D., Subramanian, S. (2020). COVID-19: Is there a reasonable alternative to a comprehensive lockdown? Ideas for India, March 28, 2020.

Roberton, T., E. D. Carter, V. B. Chou, A. Stegmuller, B. D. Jackson, Y. Tam, N. Walker. 2020. Early estimates of the indirect effects of the coronavirus pandemic on maternal and child mortality in low-and middle-income countries. Available at SSRN 3576549.

Rodrigues, Helena Sofia. 2016. Application of SIR epidemiological model: new trends. International Journal of Applied Mathematics and Informatics 10: 92-97.

Saif, S.K. 2020. Afghanistan extends lockdown amid upward COVID-19 trend. Ankara: Anadulo Agency.

Sanche, S., Y.T. Lin, C. Xu, E. Romero-Severson, N. Hengartner, and R. Ke. 2020. High contagiousness and rapid spread of severe acute respiratory syndrome coronavirus 2. Emerging Infectious Diseases 26 (7): 1470-1477.

Shiva, M. 2020. We need a better head start for the next pandemic. VoxEU.org, April 26, 2020.

Smith, L., T. Baker, G. Demombynes, P. Yadav. 2020. COVID-19 and oxygen: Selecting supply options in LMICs that balance immediate needs with long-term cost-effectiveness. Centre for Global Development.

Thompson, R.N. 2020. Epidemiological models are important tools for guiding COVID-19 interventions. BMC Medicine 18: 152. https://doi.org/10.1186/s12916-020-01628-4.

Unicef. 2020. Infographic: Get the facts on handwashing. United Nations Children's Fund. https://www. unicef.org/stories/infographic-get-facts-handwashing

Wagner, A. B., E. L. Hill, S. E. Ryan, Z. Sun, G. Deng, S. Bhadane, J. Acharya. 2020. Social distancing has merely stabilized COVID-19 in the US. medRxiv.

The World Bank Group. 2020. World development indicators. Washington: The World Bank Group. 
WHO. 2020. New WHO estimates: Up to 190000 people could die of COVID-19 in Africa if not controlled. Geneva: World Health Organisation.

WHO-China. 2020. Report of the WHO-China joint mission on coronavirus disease 2019 (COVID-19). Geneva: World Health Organisation.

Publisher's Note Springer Nature remains neutral with regard to jurisdictional claims in published maps and institutional affiliations. 